\title{
Modified and Combined Lateral and Posterolateral Approach an Alternative Treatment to Tibial Plateau Fractures
}

\author{
Gunaseelan $\mathrm{P}^{*}$, Aishah N and Hwang PX
}

Departmen of Orthopaedics and Traumatology, Hospital Sultanah Aminah Johor Bharu, Malaysia

${ }^{*}$ Corresponding author: Gunaseelan P, Departmen of Orthopaedics and Traumatology, Hospital Sultanah Aminah Johor Bharu, Malaysia, Tel: 60122114974, E-mail: gunaseelandr@gmail.com

Citation: Gunaseelan P, Aishah N, Hwang PX (2018) Modified and Combined Lateral and Posterolateral Approach an Alternative Treatment to Tibial Plateau Fractures. J Orthop Physiother 1(1): 105. doi: 10.15744/2639-930X.1.105

Received Date: February 08, 2018 Accepted Date: September 19, 2018 Published Date: September 21, 2018

\begin{abstract}
The selection of a surgical approach for the treatment of tibia plateau fractures is an important decision. Approximately $7 \%$ of all tibia plateau fractures affect the posterolateral corner. Displaced posterolateral tibia plateau fractures require anatomic articular reduction and buttress plate fixation on the posterior aspect. These aims are difficult to reach through a lateral or anterolateral approach. The standard posterolateral approach with fibula osteotomy and release of the posterolateral corner is a traumatic procedure, which includes the risk of fragment denudation. Isolated posterior approaches do not allow sufficient visual control of fracture reduction, especially if the fracture is complex. Therefore, the aim of this case report was to present a surgical approach for posterolateral tibial plateau fractures that both protects the soft tissue and allows for good visual control of fracture reduction. The approach involves a lateral arthrotomy for visualizing the joint surface and a posterolateral approach for the fracture reduction and plate fixation, which are both achieved through one posterolateral skin incision. Using this approach, we achieved reduction of the articular surface and stable fixation in patients at the final follow-up visit. No complications and no loss of reduction were observed. Additionally, the new posterolateral approach permits direct visual exposure and facilitates the application of a buttress plate. This approach does not require fibular osteotomy, and fragments of the posterolateral corner do not have to be detached from the soft tissue network.
\end{abstract}

Keywords: Tibial plateau; Fractures; Therapeutics; Internal fixators; Approach

\section{Introduction}

Articular stepoffs and angular deformities after operative treatment of tibial plateau fractures are observed in $19 \%$ to $26 \%$ of cases $[1,2]$. There is evidence in the literature that the quality of the reduction correlates with the clinical outcome $[3,4]$. This correlation is further supported by the fact that painful intra-articular malunions of the tibial plateau require complicated operations such as combined extra- and intra-articular osteotomies to improve their outcome [5,6]. These results indicate that an anatomic fracture reduction without intra-articular stepoffs is an important goal in the treatment of tibial plateau fractures $[2,4,6]$. At least $7 \%$ of all tibia plateau fractures lie in the region of the posterolateral corner [2]. Fractures in this region usually cannot be adequately treated by using a lateral or anterolateral approach $[7,8]$. The fragments are often covered by the fibula head and the ligamentous structures in the corner region of the popliteus muscle; hence, they are difficult to reduce and fix [1]. To minimize this problem, a posterolateral approach was developed by Lobenhoffer et al. [5] with this approach, a fibular osteotomy and detachment of the joint capsule and meniscotibial ligaments from the lateral tibia plateau allow for the exposure and examination of the posterolateral joint surfaces of the tibia. However, in addition to the fibular osteotomy, dissection of the posterolateral structures and ligaments was necessary in this instance [6]. According to our own experience, Lobenhoffer's approach [5] provides a good view of the posterolateral corner of the tibia plateau. However, this strategy may lead to relatively extensive trauma of the soft tissue of the posterolateral corner. Isolated posterior approaches allow for fragment fixation [1,9], but the visual control of fracture reduction is limited, especially if the fractures are more complex. Therefore, in this case report, a modified surgical technique for the treatment of posterolateral tibia plateau fractures is introduced. This technique includes a posterolateral approach for fracture reduction and fixation combined with a lateral arthrotomy to visually control the fracture alignment and the joint surface. A case example is presented, to show posterolateral approch can be an alternative treatment for posterior lateral column fracture of the tibia plateau fracture.

\section{Case Report}

18 years old lady, who had a fall from 3 meter height with left foot on the ground first, followed by kneeling position with an 
outstretch hand, complain of right knee, right wrist and left ankle pain to the casualty. Case was referred to the orthopedic team oncall for further management. Right knee was swollen with no evidence of neurovascular injury. X-rays workout noted she sustained undisplaced left talus neck closed fracture, right radius distal end extra articular closed fracture, and right tibial plateau closed fracture with posterolateral fragments. Right knee CT scan was proceeded to facilitate preoperative planning. After mutual discussion regarding diagnosis and options of operation, surgery was scheduled electively for open reduction internal fixation of the right distal radius and the right tibial plateau fracture while left talus was managed nonsurgically. Perioperatively, floppy lateral position was used to facilitate access to both posterolateral and anterolateral aspect of tibial plateau. Skin incision was marked central at upper pole of head of fibula, curve proximal posteriorly along lateral border of biceps femoris, distally following convential anterolateral approach to tibial plateau. Common peroneal nerve was isolated and protected; an intermuscular window was created between biceps femoris and lateral head gastrocnemius. Distally the window was limited by the soleus muscles which could be dissected to allow sitting of a plate, while medially it is limited by popliteal artery. A second window with lateral arthrotomy with submeniscus approach allowed direct visualization of anterolateral aspect. Fragments reduction achieved with preliminary k-wires fixation, articular surfaces restored, posterolateral fragment supported by a buttress plate, while larger anterolateral fragment was fixed with a conventional anterolateral proximal tibia plate. Postoperative, passive continous motion machine was applied with adequate analgesia coverage. Patient was discharged well and subsequently followed by at 2 weeks/ 6 weeks/ 3 months/ 6 months. During 6 month post op outpatient visit, she reported no pain over right knee. Right knee ligaments was stable, 0-120 knee range of motion achieved, with no joint line tenderness (Figure 1,2,3 and 4).

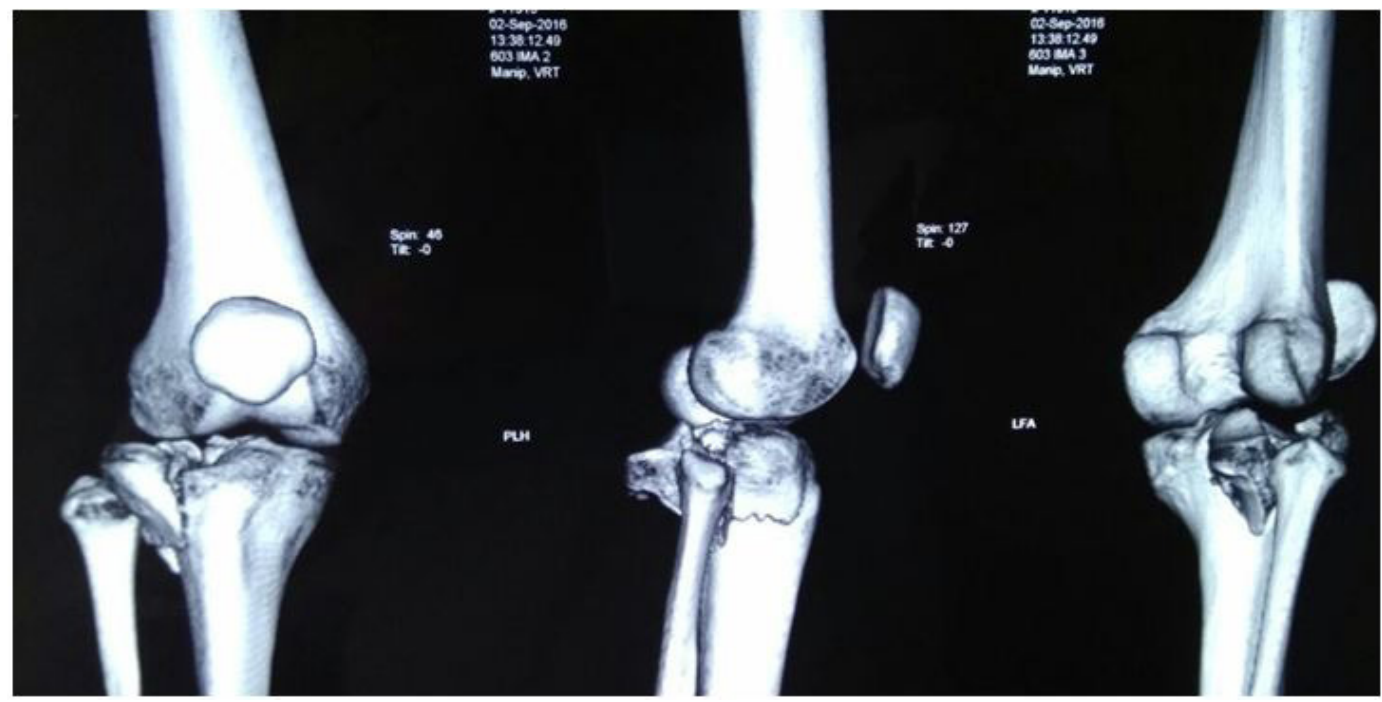

Figure 1: 3D reconstruction Images: anterolateral larger fragment and smaller posterolateral fragment

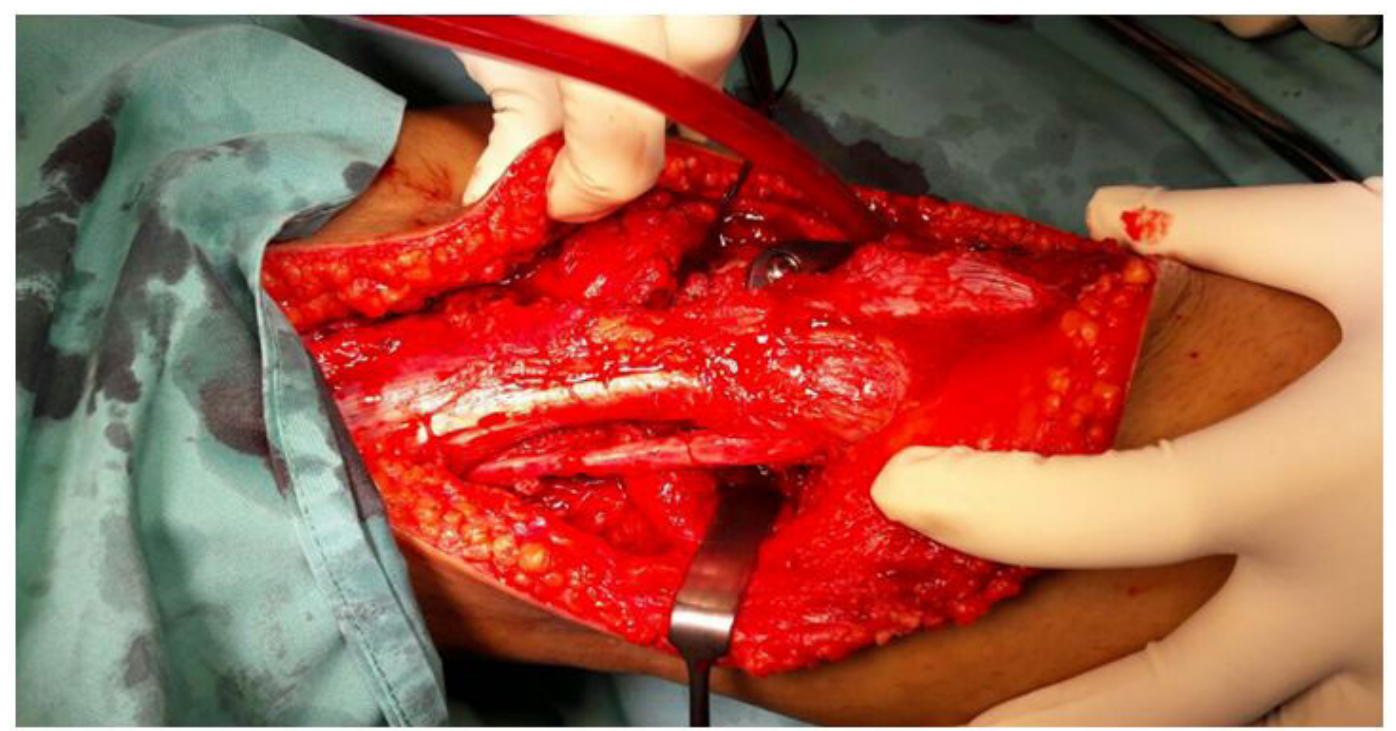

Figure 2: 2 windows utilized (with suction yankauer pointed and retractor pointed), common peroneal nerve isolated next to biceps femoris. 2 plates applied addressing the two separate fragments. Lateral arthrotomy (edge of meniscus stay suture tied) allows accurate articular reduction 


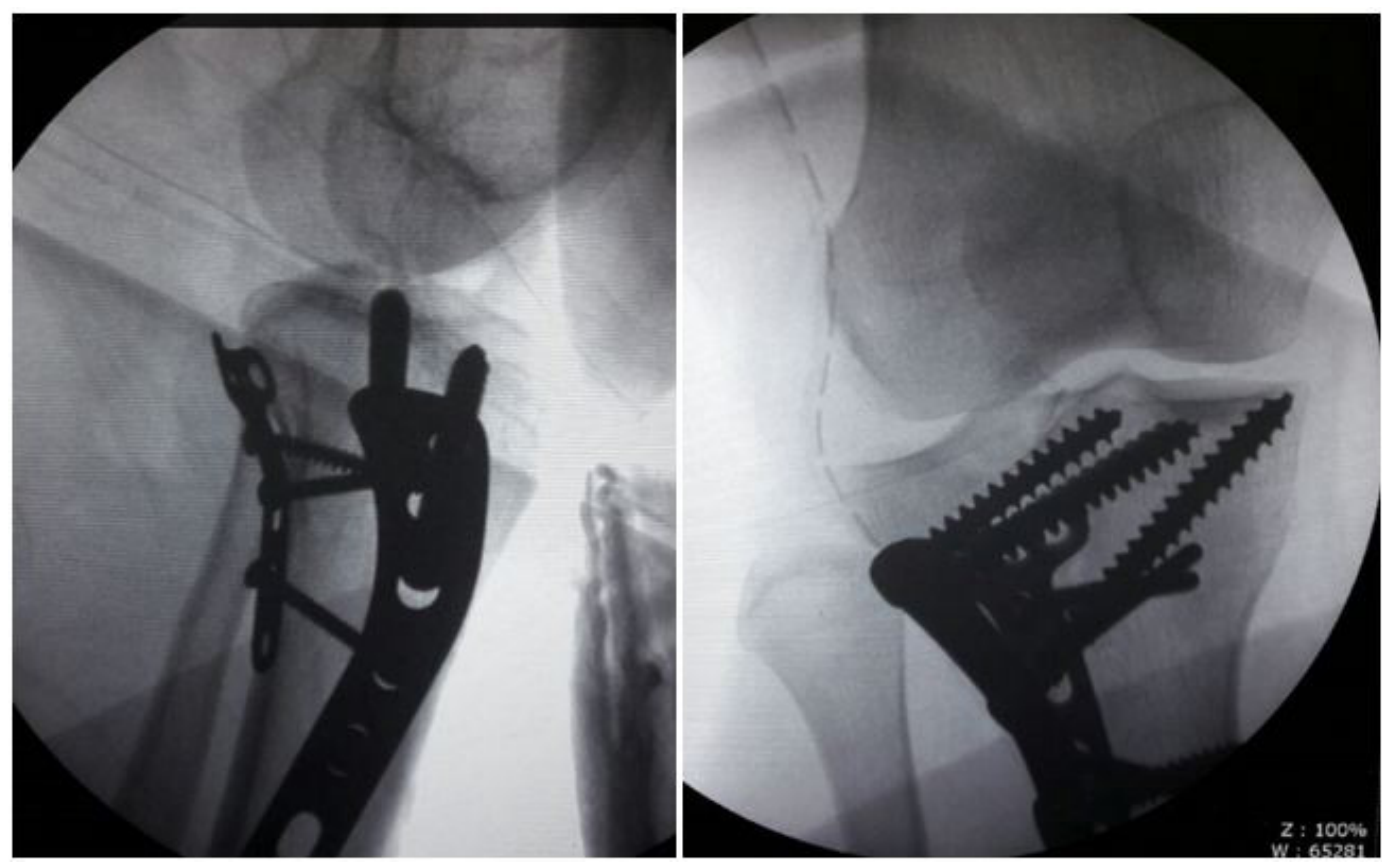

Figure 3: Intraop Image intensifier images with reduced fracture

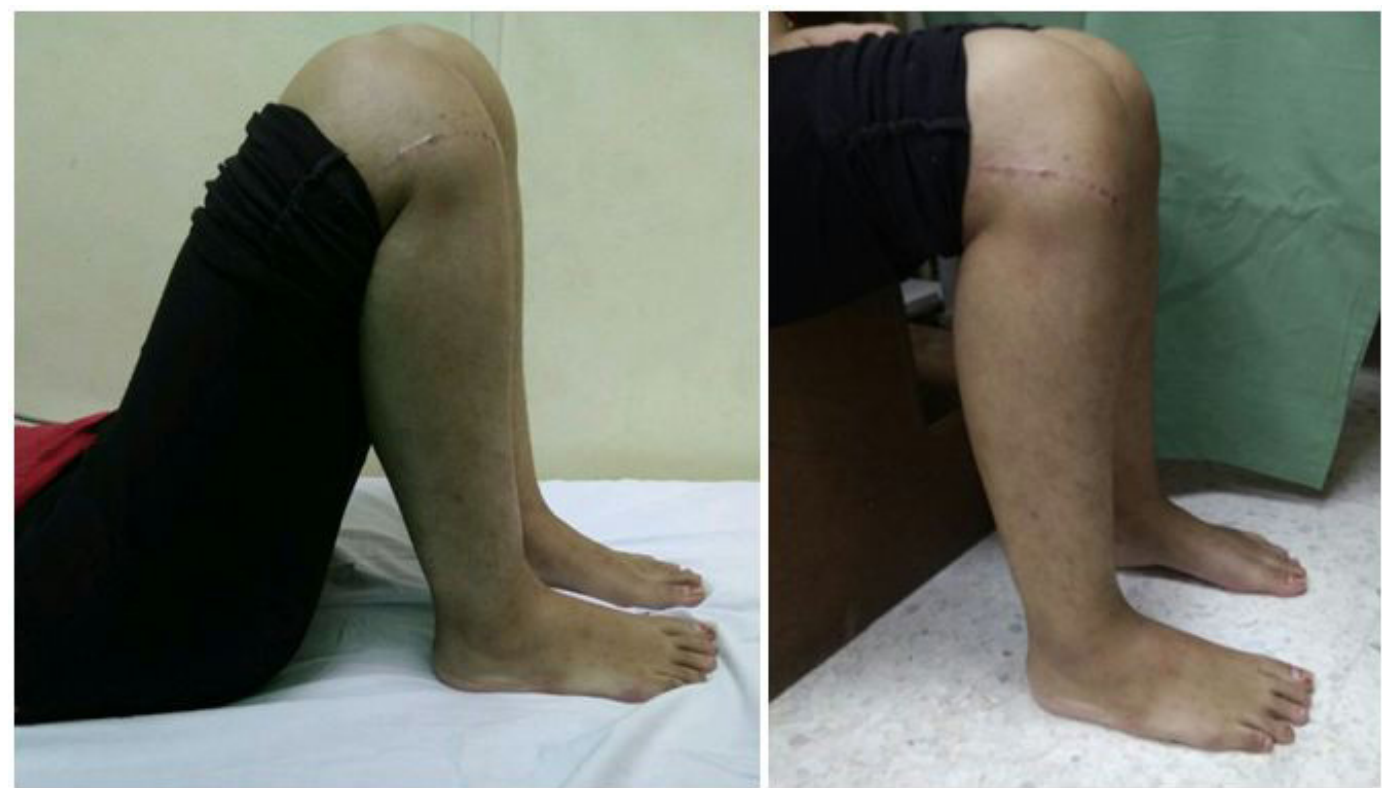

Figure 4: Post op 6 month: Right knee achieved full range of motion

\section{Discussion}

In recent years, three-dimensional imaging has contributed enormously to the improvement of the operative treatment of joint fractures. In particular, preoperative fracture analysis allows surgeons to precisely plan their approach and treatment strategies $[10,11]$. Specifically, on the tibia plateau, the dorsal parts of the plateau are generally unsatisfactorily dealt with by the wellestablished medial and lateral standard access strategies [12]. Therefore, multiple authors recommend that tibial plateau fractures involving the posteromedial region should be treated using posteromedial approaches $[2,4,7,12]$. Barei et al. demonstrated that $74 \%$ of bicondylar tibial plateau fractures involve the posteromedial parts [11]. For this type of fracture, they used an anterolateral and a posteromedial standard approach. Although posterolateral and posteromedial tibial head fractures occur with similar frequency [6], the posterolateral approach is used less frequently than the posteromedial approach [2]. The posterolateral approach described by Lobenhoffer et al. [6] allows an optimal overview of the posterolateral tibia plateau. However, in our opinion, the disadvantage of Lobenhoffer's approach is the considerable trauma to the soft tissue caused by extended exposure, particularly to the posterolateral structures. The loss of reduction observed in $50 \%$ of cases with tibia plateau fractures treated with this approach [6] could also be caused by trauma to the posterolateral corner soft tissue and the associated reduced blood circulation in the fragments. Thus, a good view and exposure of the posterolateral fragments might be gained but at the expense of tissue nutrition. In our opinion, the isolated posterior approaches to the posterolateral corner [7-9,12] have some disadvantages compared with 
the approach outlined here. With dorsal approaches, a dorsal arthrotomy in the posterolateral area is necessary. In this area, strong and important ligaments like the ligamentum popliteum obliquum, ligamentum popliteum arcuatum, popliteal tendon, joint capsule, ligamentum meniscotibiale, and others have to be dissected. With our approach, these ligaments can be preserved. We laterally dissected the capsule and the ligamentum meniscotibiale to expose the entire lateral tibial plateau. Therefore, the damage to the soft tissue might be even higher with the isolated posterior approaches compared with our approach. In particular, the peroneal nerve needs to be exposed in posterior approaches in a similar manner [1,12] as described here. Tao et al. [1] recently introduced a modified L-shaped incision to expose the posterior aspect of the lateral plateau through the intervals among the medial gastrocnemius, lateral gastrocnemius, popliteus, and soleus. Although blunt dissection among muscle intervals is preferred, exposure and retraction of the popliteal neurovascular bundle is unnecessary, tedious, and risky. Avery similar approach to the dorsal parts of the proximal tibia was presented by Zhang et al. [12] A disadvantage of this technique is that it is performed in the prone position [12]. By using the isolated posterior approaches [7,9,12], only a limited view of the lateral plateau is possible. It is also very difficult to extend isolated dorsal approaches, especially when the fractures are more complex and involve the lateral or anterolateral parts of the tibia plateau, as seen in the patient presented here. Therefore, the presented approach is performed in a lateral position. For the modified posterolateral approach that is presented here, visual control of the reduction of the fracture is achieved through a conventional, lateral standard arthrotomy, which is accomplished through the same skin incision. The anatomic reduction of the fracture and internal fixation are performed from the dorsal side. Therefore, as a result of the modified posterolateral approach, the fragments are not denuded. In the event of complex damage to the posterolateral corner, the modified posterolateral approach described here can be extended, if necessary, by additional fibular osteotomy, as described by Lobenhoffer [5]. Damage to the upper tibiofibular joint and nerve injuries caused by an oscillating saw blade can be avoided by using the presented technique. Nevertheless, peroneal nerve injuries can occur as a result of manipulation during surgery. Nerve injuries were not observed in our patient. A limitation of the presented modified posterolateral approach is that it cannot be extended distally because of the trifurcation vessels that traverse the interosseous membrane approximately $5 \mathrm{~cm}$ below the joint line [12]. However, because the lateral tibial metaphysis has a posterior inclination angle of approximately 45 deg [12] and the posterolateral split fracture segment is usually less than $4 \mathrm{~cm}$ in cortical length [12], this limitation does not seem to be a problem in practice. Therefore, iatrogenic injuries of blood vessels did not occur in our case. To avoid such possible injuries, it is very important that the posterolateral plate is not placed too far distally, that the popliteal artery is carefully dissected, and that the plate is positioned under visual control. When inserting the plate, it is also important that the distal end of the plate has continuous contact with the tibial bone so that the vessel cannot extend under the plate. Another disadvantage of the presented operation technique is that it can be difficult to address additional medial plateau fractures when the patient is lying in the described lateral position. In case of additional involvement of the medial plateau, we either recommend to intraoperatively switch the patient from a lateral to a supine position or to use Lobenhoffer's approach, which can be performed in the supine position and allows an additional medial approach without changing the patient's intraoperative position. Precise knowledge of the anatomy of the posterolateral corner is required to perform the presented approach because nerves and vessels that border the operation site and almost encircle this area must be dissected and separated from one another. To avoid damage to vessels and nerves, the surgery must be done very carefully.

\section{Conclusion}

The modified and combined lateral and posterolateral approach to the tibia plateau presented here has not been previously described in the literature. This approach could potentially be used to treat posterolateral tibia plateau fractures. The modified posterolateral approach demands precise knowledge of the anatomic structures of that region. The advantages of the presented modified posterolateral approach are the protection of soft tissue and ligamentous structures, the ability to dispense with fibular osteotomy, and the preservation of soft tissue around the posterolateral fragments.

\section{References}

1. Frosch KH, Balcarek P, Walde T, Stürmer KM (2010) A new posterolateral approach without fibula osteotomy for the treatment of tibial plateau fractures. J Orthop Trauma 24: 515-20.

2. Carlson DA (2005) Posterior bicondylar tibial plateau fractures. J Orthop Trauma 19: 73-8.

3. Ruedi TP (2007) AO-Principles of Fracture Management ( $2^{\text {nd }}$ Edn) New York: Thieme, USA.

4. Li Q, Zhang YQ, Chang SM (2014) Posterolateral fragment characteristics in tibial plateau fractures. Int Orthop 38: 681-2.

5. Lobenhoffer P, Gerich T, Bertram T, Lattermann C, Pohlemann T, et al. (1997) Particular posteromedial and posterolateral approaches for the treatment of tibial head fractures. Unfallchirurg 100: 957-67.

6. Solomon LB, Stevenson AW, Baird RP, Pohl AP (2010) Posterolateral transfibular approach to tibial plateau fractures: technique, results, and rationale. J Orthop Trauma 24: 505-14.

7. Tao J, Hang DH, Wang QG, Gao W, Zhu LB, et al. (2008) The posterolateral shearing tibial plateau fracture: treatment and results via a modified posterolateral approach. Knee 15: 473-9.

8. Chang SM, Zheng HP, Li HF, Jia YW, Huang YG, et al. (2009) Treatment of isolated posterior coronal fracture of the lateral tibial plateau through posterolateral approach for direct exposure and buttress plate fixation. Arch Orthop Trauma Surg 129: 955-62.

9. Pierrie SN, Harmer LS, Karunakar MA, Angerame MR, Andrews EB, et al. (2016) Limited added value of the posterolateral approach. J Knee Surg 29: 21-7.

10. Pires RES, Giordano V, Wajnsztejn A, Oliveira Santana E Junior, Pesantez R, et al. (2016) Complications and outcomes of the transfibular approach for posterolateral fractures of the tibial plateau. Injury 47: 2320-5. 
11. Yu B, Han K, Zhan C, Zhang C, Ma H, et al. (2010) Fibular head osteotomy: a new approach for the treatment of lateral or posterolateral tibial plateau fractures. Knee 17: 313-8.

12. Chen WT, Zhang YQ, Chang SM (2013) Posterolat-7eral approach for plating of tibial plateau frac-tures and the risk of injury to the anterior tibial vessels. J Orthop Trauma 27: e228-9. 